\title{
Morfología de Dasylirion cedrosanum y su correlación con imágenes en localidades del Sureste de Coahuila
}

\section{Morphology of Dasylirion cedrosanum and its correlation with images in localities of Southeastern Coahuila}

\section{Emir Lenin Serafín-Higuera (C) Adriana Antonio-Bautista ${ }^{2}$ C] Adalberto Benavides- Mendoza 3 [}

${ }^{1}$ Doctorado en Recursos Fitogenéticos para Zonas Áridas, Universidad Autónoma Agraria Antonio Narro, Calzada Antonio Narro No. 1923, CP. 25315, Buenavista, Saltillo, Coahuila, México.

${ }^{2}$ Cinvestav unidad Saltillo, Programa de Sustentabilidad de los Recursos Naturales y Energía, Avenida Industria Metalúrgica No.1062, CP. 25900, Parque Industrial Saltillo-Ramos Arizpe, Ramos Arizpe, Coahuila, México.

${ }^{3}$ Departamento de Horticultura, Universidad Autónoma Agraria Antonio Narro, Calzada Antonio Narro No. 1923, CP. 25315, Buenavista, Saltillo, Coahuila, México.

*Autor de correspondencia:

abenmen@gmail.com

\section{Nota científica}

Recibida: 17 de noviembre 2021

Aceptada: 24 de enero 2022

Como citar: Serafín-Higuera EL, Antonio-Bautista A, BenavidesMendoza A (2022) Morfología de Dasylirion cedrosanum y su correlación con imágenes en localidades de Sureste de Coahuila. Ecosistemas y Recursos Agropecuarios 9(1): e3198. DOI: 10.19136/era.a9n1.3198
RESUMEN. El procesamiento digital de imágenes es una herramienta que facilita el análisis morfológico. Por lo que, se planteó evaluar las características morfológicas de plantas de sotol usando imágenes digitales. Para esto, se tomaron las variables: altura de la planta, diámetro de cobertura, altura y diámetro del tallo de 10 plantas en cuatro localidades de Coahuila. Además, de fotografías a cada espécimen y se analizaron los datos morfológicos en el programa ImageJ. Utilizando un análisis de regresión lineal y de correlación se realizó una comparación entre los datos medidos de forma manual y digital. Los resultados muestran que las variables determinadas de forma manual tienen correlación positiva y significativa con las variables de las fotografías analizadas digitalmente. El uso de fotografías y del análisis digital es una herramienta de apoyo para caracterizar la morfología y seleccionar las plantas de sotol.

Palabras clave: Aprovechamiento sustentable, conservación, ImageJ, producción, sotol.

ABSTRACT. Digital image processing is a tool that facilitates morphological analysis. Therefore, it was proposed to evaluate the morphological characteristics of sotol plants using digital images. For this purpose, the following variables were taken: plant height, cover diameter, height and stem diameter of 10 plants in four localities of Coahuila. In addition, photographs were taken of each specimen and the morphological data were analyzed in the ImageJ program. Using linear regression and correlation analysis, a comparison was made between manually and digitally measured data. The results show that the manually determined variables are positively and significantly correlated with the variables of the digitally analyzed photographs. The use of photographs and digital analysis is a support tool to characterize the morphology and select the sotol plants.

Key words: Sustainable harvesting, conservation, ImageJ, production, sotol. 


\section{INTRODUCCIÓN}

Las plantas de sotol (Dasylirion cedrosanum) son un componente importante de las regiones áridas del centro y norte de México (Encina-Domínguez et al. 2013). Actualmente se utiliza para la elaboración de la bebida destilada llamada sotol (ReyesValdés et al. 2019), siendo los estados de Coahuila, Chihuahua y Durango los que cuentan con la denominación de origen para su elaboración (IMPI 2002). En los últimos años la producción del destilado de sotol ha cobrado interés y ha generado una demanda creciente (Reyes-Valdés et al. 2012). En el 2005 el ingreso por la venta de esta bebida fue de alrededor de 19.5 millones, mientras que para el 2008 se registraron 58.2 millones de pesos (Becerra-López et al. 2020).

El estado de Coahuila tiene poblaciones naturales de sotol, pero no se tienen plantaciones forestales comerciales y la materia prima para producir la bebida proviene de poblaciones silvestres (Flores-Gallegos et al. 2019). Con respecto a las autorizaciones en materia de aprovechamiento de recursos forestales no maderables emitidos por la Secretaría de Medio Ambiente y Recursos Naturales (SEMARNAT), se indica que se deben llevar a cabo estudios técnicos justificativos para la extracción de la planta en poblaciones naturales, por lo que se requiere un inventario con la caracterización morfológica de los individuos del sitio a aprovechar (Reyes-Valdés et al. 2019). Por tal motivo, la caracterización de individuos o poblaciones es fundamental para realizar los inventarios de especies distribuidas naturalmente, sobre todo de aquellas que cumplen con las características para ser aprovechables (Cano et al. 2011).

En el caso del sotol la forma más común de realizar los inventarios y obtener información de las plantas es siguiendo la metodología del análisis multidimensional y tablas de producción (Cano et al. 2005). Con la finalidad de saber si el sitio contiene plantas que cumplen con las dimensiones para su aprovechamiento, se deben llevar a cabo dichos inventarios tomando en cuenta las dimensiones de las plantas (SEMARNAT 1997). En la actualidad, el procesamiento digital de imágenes es una herramienta que facilita el análisis morfológico de una gran cantidad de plantas en un periodo relativamente corto (Li et al. 2020). Es una herramienta útil en los estudios de crecimiento y calidad de plantas (Zhang. 2020, Gupta et al. 2021), área foliar (Kumar et al. 2019), biomasa (Li et al. 2020), inventarios forestales (Acosta et al. 2017), características morfológicas de semillas (Perea-Flores et al. 2011, Lallana et al. 2018), detección de enfermedades y de condiciones de estrés (Abbas et al. 2021, Veerendra et al. 2021). Bajo la hipótesis de que el uso del proceso de imágenes permitirá obtener información correlacionada con la información obtenida a partir de mediciones manuales, se planteó el objetivo de evaluar las características morfológicas de plantas de sotol a través del uso de imágenes digitales en cuatro localidades del sureste de Coahuila.

\section{MATERIALES Y MÉTODOS}

El área de estudio se ubica en el estado de Coahuila. Comprendió las localidades de Buenavista, San Lorenzo, General Cepeda y Cañón del Tule (Figura 1). Entre sus características biofísicas Buenavista presenta vegetación secundaria, suelos leptosoles y calcisoles, tipo de clima árido semicálido (BSohw) y en San Lorenzo semiárido-templado (Bs1kw) con bosque de pino perturbado. En cambio, en General Cepeda el tipo de vegetación que predomina es matorral rosetófilo, los suelos principales son leptosol, con un tipo de clima árido semicálido (BSohw) y en el Cañón del Tule los suelos principalmente son leptosoles, el clima es árido semicálido (BSohw) y el tipo de vegetación es matorral desértico rosetófilo (CONABIO 2001). En cada localidad se utilizó un muestreo de punto central por cuadrante que consistió en localizar una serie de puntos aleatorios dentro del sitio a muestrear, el área de cada punto localizado se dividió en cuatro partes iguales o cuadrantes y se tomaron datos de las plantas localizadas en cada cuadrante (Cano et al. 2011). 


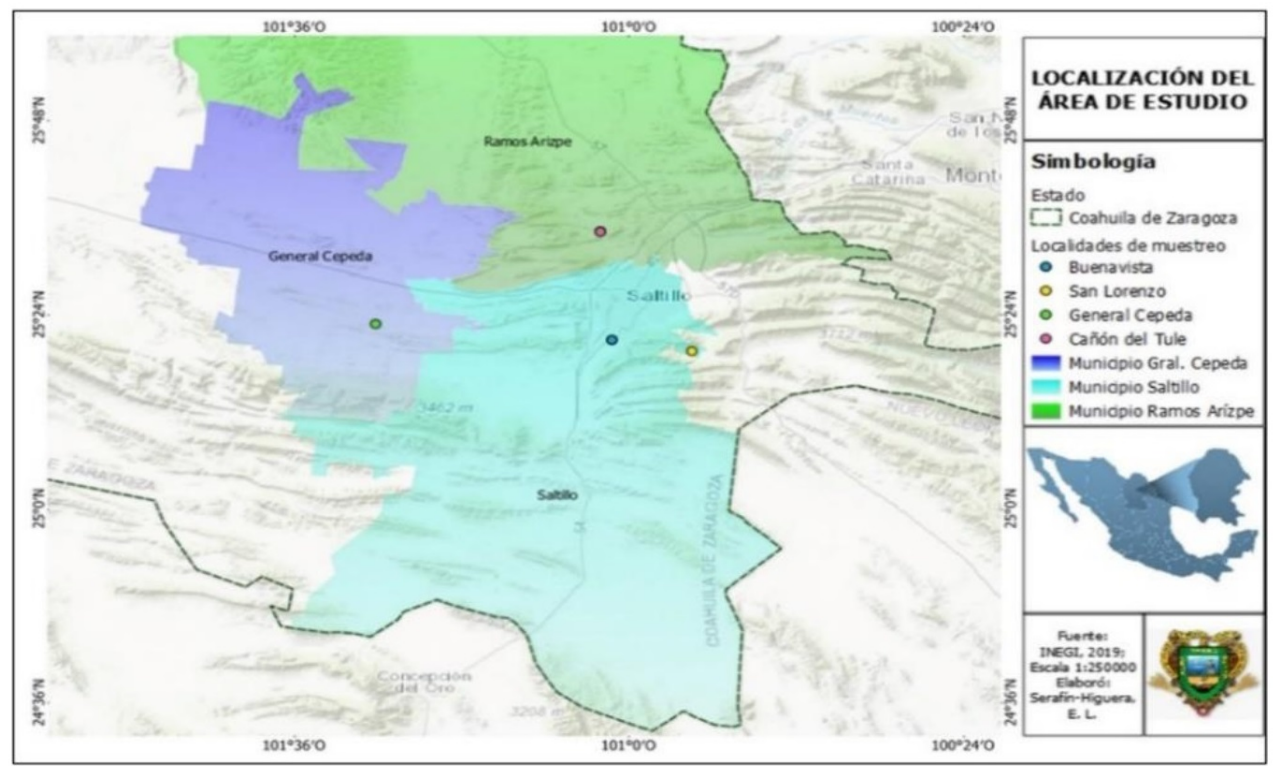

Figura 1. Localización del área de estudio.

\section{Toma de datos morfológicos en campo}

Durante los meses de junio y julio del 2021, se tomaron datos de 10 plantas simples (una yema) de $D$. cedrosanum en cada una de las cuatro localidades de estudio. Se consideraron plantas de poblaciones silvestres con diferentes estadios de desarrollo. Además, se colectaron muestras de ejemplares que se herborizaron y que se encuentran en la colección del herbario Antonio Narro Saltillo México (ANSM) con números de registros 102850, 102851, 102852 y 102853.

Los datos morfológicos tomados en campo fueron: altura de planta (AP), diámetro de cobertura (CR), altura (AT) y diámetro del tallo (DT) de acuerdo con la metodología de Cano et al. (2005). Para lo cual con un flexómetro graduado en $\mathrm{cm}$ se midió la altura de la planta desde la base hasta la parte alta de la corona, el diámetro de cobertura se midió en la parte superior de la corona obteniendo el promedio de dos medidas perpendiculares, la altura del tallo se cuantificó a partir de la base del suelo hasta donde termina el tallo o la base del cogollo central y con el uso de una forcípula de aluminio modelo Haglof Sweden s-882-00 se midió el diámetro del tallo en el eje central a una altura de $30 \mathrm{~cm}$.

\section{Fotografías tomadas en campo}

La toma de fotografías se realizó considerando las variables morfológicas de interés, las herramientas digitales y el espacio físico en donde se encontraban las plantas. La toma de fotografías se realizó en un ambiente abierto de las 11:00 a las 13:00 h, para lo cual se tomaron dos fotografías a cada espécimen. De la base del tallo de la planta al tripié de la cámara (altura $1.10 \mathrm{~m}$ ) se consideró una distancia de $3 \mathrm{~m}$, la fotografía se tomó con una cámara digital marca SONY modelo DSC-W830 de 20.1 mega pixeles. Al momento de la captura no se seleccionó ningún nivel de acercamiento y se desactivo la opción de flash. Para cada toma fotográfica, a un costado de la planta, se colocó una escala de referencia con dimensiones de $50 \times 20 \mathrm{~cm}$.

\section{Procesamiento de imágenes}

El análisis de imágenes se realizó con el software libre ImageJ versión 1.52a (Ferreira et al. 2018). Para el despliegue de las fotografías se utilizó el comando del menú del programa. El proceso de calibración de las imágenes consistió en determinar una medida de referencia, para lo cual con la herramienta de selección de líneas rectas (Straight) se dibujó una línea a lo largo de la escala, después en la barra de 
herramientas se seleccionó la opción Analize y Set scale en el cual se indicó una distancia de $50 \mathrm{~cm}$ (altura de la escala). Las mediciones se realizaron con las herramientas de lupa (Magnifying glass) y de selección de líneas rectas (Straight). La altura de la planta (AP. ImageJ) se midió dibujando una línea vertical en la parte media entre el límite del suelo y la base de la planta hasta la parte más alta de la corona. El diámetro de la cobertura (CR. ImageJ) se estimó partiendo de la parte media de la corona, dibujando una línea horizontal hacia los extremos de las puntas de las hojas. Para la altura del tallo (AT. ImageJ) se dibujó una línea vertical entre el límite superior del suelo hasta el límite final del tallo o la base del cogollo central. El diámetro del tallo (DT. ImageJ) se estimó dibujando una línea horizontal en la parte central, abarcando los bordes de la corona. La sección longitudinal (SL. ImageJ) se obtuvo con la herramienta de polígonos para la cual se delimitó el contorno de la cobertura foliar. Para obtener todas las medidas se utilizaron los comandos Analyze y Measure. Las unidades utilizadas para las variables altura de la planta, diámetro de cobertura, altura y diámetro del tallo fueron centímetros $(\mathrm{cm})$, mientras que para la sección longitudinal se utilizó centímetros cuadrados $\left(\mathrm{cm}^{2}\right)$.

\section{Análisis estadístico}

Se realizó un análisis de regresión lineal simple para las variables medidas de forma manual en campo y analizadas con el programa ImageJ. La utilidad de los modelos se corroboró con una prueba de Fisher y la validación se hizo por medio de análisis de residuales. La prueba de Shapiro Wilk se utilizó para la normalidad y para la homocedasticidad la prueba de Breush-Pagan (Breusch y Pagan 1979). Además, se llevó a cabo un análisis de correlación. Para ello se utilizó el coeficiente de correlación de Pearson, una prueba de t para analizar la significancia de la correlación $(p<0.05)$ y de distribución de normalidad (Cooksey 2020). El análisis se llevó a cabo en el programa $R^{\circledR}$.

\section{RESULTADOS Y DISCUSIÓN}

\section{Análisis de correlación y de regresión entre medi- ciones manuales y digitales}

De acuerdo con la Tabla 1 todas las variables medidas en campo y las variables analizadas digitalmente se correlacionaron significativamente $(p<$ $0.05)$ con coeficientes altos. El análisis de correlación de la variable SL. ImageJ con las variables obtenidas en campo (Tabla 2) muestra que los coeficientes de correlación no son altos. La correlación con la variable CR. ImageJ fue la más alta y con AT y AT. Image J fueron bajas y no significativos ( $p$ 0.07) y ( $p$ 0.09). Por lo tanto, el uso de la variable SL. ImageJ para la predicción de los valores de las variables obtenidas en campo y analizadas en imágenes puede no ser adecuado. De acuerdo con el análisis de regresión lineal simple (Figura 2), las comparaciones de las variables que se midieron manualmente con las analizadas digitalmente presentaron altos coeficientes de determinación, lo que indica que los modelos son fiables y que hay relación entre las variables.

Tabla 1. Resultados de correlaciones de mediciones manuales vs digitales.

\begin{tabular}{cccc}
\hline Variable & Variable & Coeficiente de correlación & $P$ \\
\hline AP & AP. ImageJ & 0.80 & 0.00 \\
CR & CR. ImageJ & 0.89 & 0.00 \\
AT & AT. ImageJ & 0.90 & 0.00 \\
DT & DT. ImageJ & 0.87 & 0.00 \\
\hline
\end{tabular}

Tabla 2. Análisis de correlación de la variable SL. ImageJ vs. variables.

\begin{tabular}{lccc}
\hline Variable & Variable & Coeficiente de correlación & $P$ \\
\hline SL. ImageJ & AP & 0.59 & 0.00 \\
SL. ImageJ & AP. ImageJ & 0.57 & 0.00 \\
SL. ImageJ & CR & 0.55 & 0.00 \\
SL. ImageJ & CR. ImageJ & 0.65 & 0.00 \\
SL. ImageJ & AT & 0.26 & 0.09 \\
SL. ImageJ & AT. ImageJ & 0.28 & 0.07 \\
SL. ImageJ & DT & 0.56 & 0.00 \\
SL. ImageJ & DT. ImageJ & 0.54 & 0.00 \\
\hline
\end{tabular}

Se han aplicado técnicas de caracterización basadas en imágenes para medir la altura de la planta de sorgo, las cuales están altamente correlacionadas con las mediciones manuales, por lo que, 
Gráfico 1. Altura de la planta (AP) vs Altura de la planta analizada con el software ImageJ (AP. ImageJ)

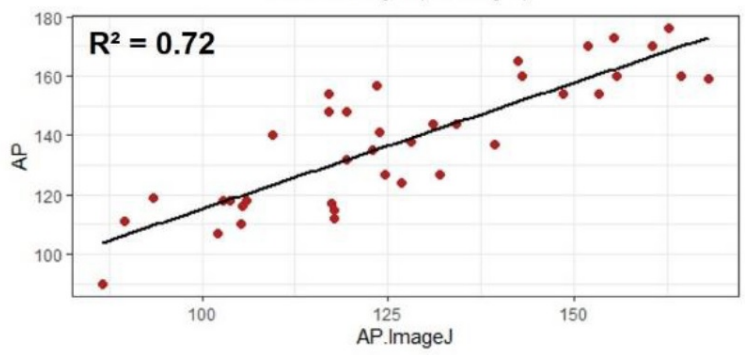

Gráfico 3. Altura del tallo (AT) vs Altura del tallo analizado con el software ImageJ (AT. Image J)

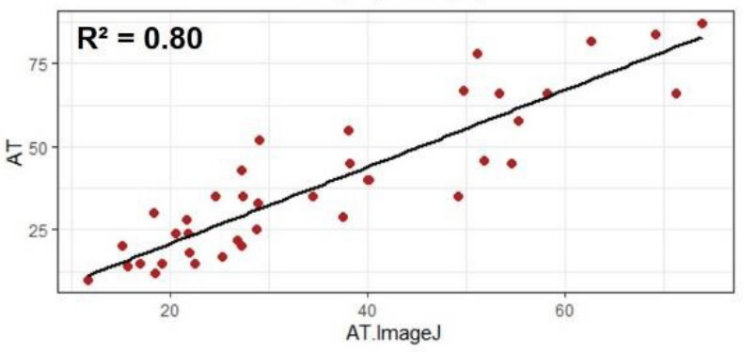

Gráfico 2. Cobertura de roseta (CR) vs Cobertura de roseta analizada con el software ImageJ (CR. Image J)

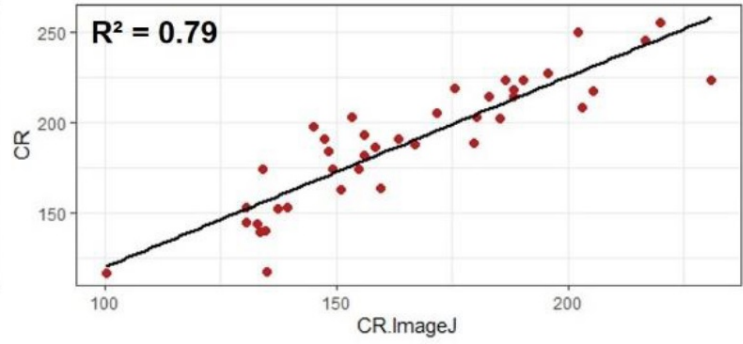

Gráfico 4. Diámetro del tallo (DT) vs Diámetro del tallo analizado con el software ImageJ (DT. Image J)

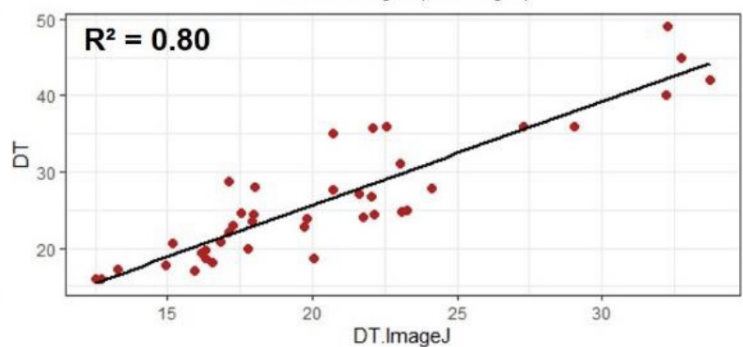

Figura 2. Resultados del análisis de regresión lineal entre las mediciones obtenidas en campo y las registradas digitalmente.

las aplicaciones de estas técnicas fueron útiles y más rápidas para medir esta variable (Wang et al. 2018). De la misma manera, Hassan et al. (2019) indican que la toma de fotografías a partir de vehículos aéreos no tripulados y su análisis constituye una herramienta eficaz para tener estimaciones rápidas de la altura de plantas de trigo. Mientras que Ma et al. (2019) proporcionan información de altos coeficientes de correlación de las medidas de altura de la planta (0.98), diámetro del tallo (0.89) y amplitud del dosel (0.95) tomadas a partir de un escáner portátil con datos medidos manualmente en la planta de maíz y consideran que el uso de estas técnicas es un apoyo importante para el fenotipado de poblaciones de plantas. Otra investigación respecto al maíz es la utilización del software Maize-IAS (Software de análisis de imágenes de maíz) para medir variables morfológicas durante el crecimiento del maíz. Dicho software es una herramienta útil y eficiente para la investigación de plantas basadas en imágenes, permite tareas automatizadas con menos mano de obra de registro, medición y el análisis cuantitativo de un gran conjunto de datos (Zhou et al. 2021). Por lo que el uso de la imagen digital permite obtener información para evaluar simultáneamente diferentes parámetros morfológicos (Kolhar y Jagtap. 2021). De acuerdo con Li et al. (2020), la técnica de análisis de imágenes se utiliza para obtener mediciones, lo cual permite maximizar la productividad, disminuir el desperdicio de recursos y cosechar los cultivos a tiempo en la producción comercial.

La Figura 3, muestra que las variables AP y AP. ImageJ se correlacionaron de mejor manera con AT, AT. ImageJ, DT y DT. ImageJ. Las variables CR y CR. ImageJ se correlacionaron positivamente con DT y DT. ImageJ a diferencia de las correlaciones con AT y AT. ImageJ que fueron no significativas $(p>$ 0.05). Los rasgos morfológicos eventualmente determinan el rendimiento y la biomasa de las plantas, lo cual es útil para conocer su aptitud y valor económico (Brophy et al. 2018). Por ejemplo, conocer los componentes morfológicos del maíz como la altura de la planta, la mazorca y la resistencia del tallo pueden facilitar la cosecha mecánica (Wei et al. 2018). En el caso del sotol, los productores buscan características morfológicas óptimas en las plantas, con la finalidad de obtener un mejor rendimiento en la producción de sotol. Por ejemplo, que tengan rosetas grandes, 


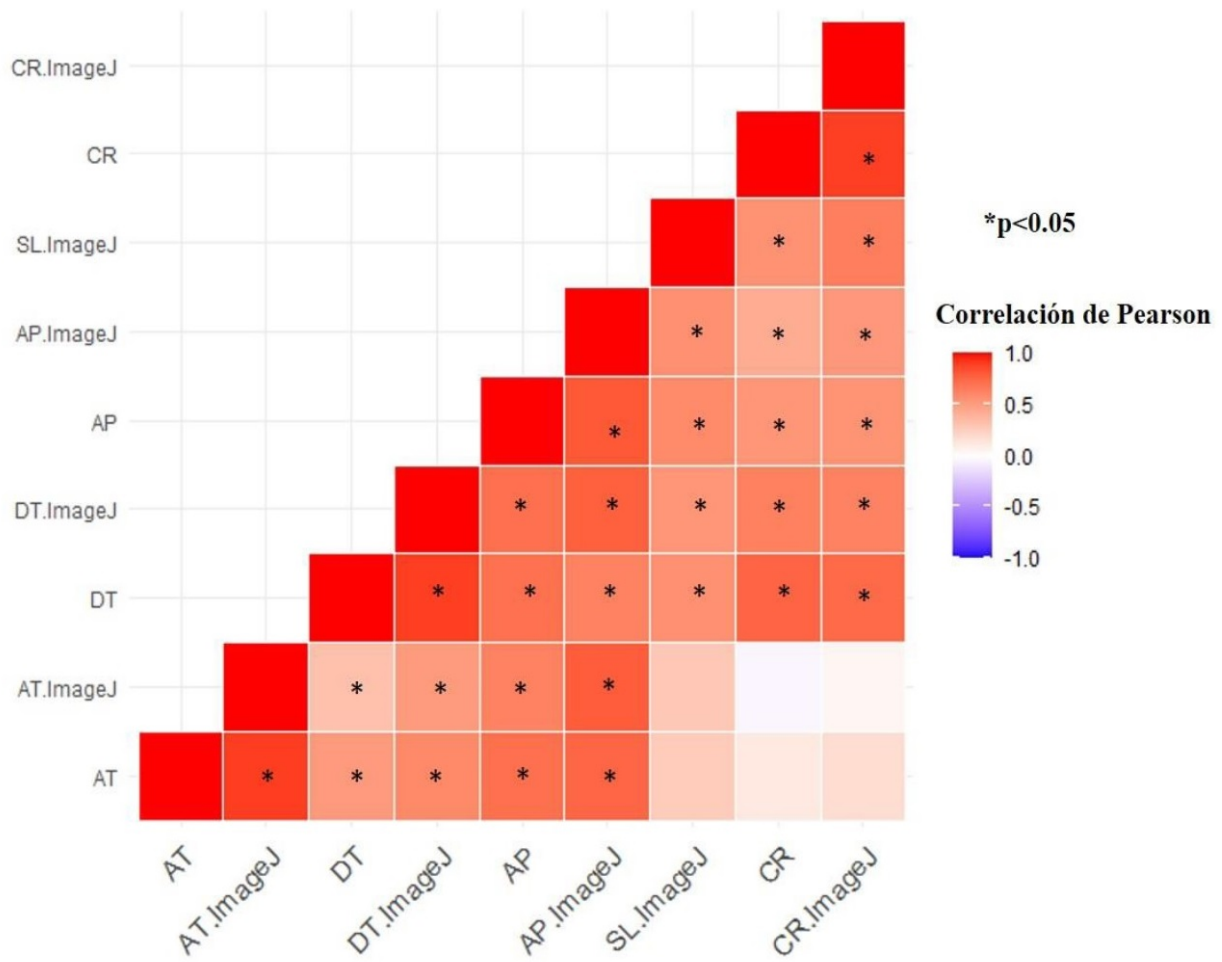

Figura 3. Correlación del conjunto de variables. Altura de la planta (AP), cobertura de roseta (CR), altura (AT) y diámetro de tallo (DT). Altura de la planta analizada con ImageJ (AP. ImageJ), cobertura de roseta analizada con ImageJ (CR. ImageJ), altura y diámetro de tallo analizado con ImageJ (AT. ImageJ, DT. ImageJ).

piñas (tallos) mayores a $30 \mathrm{~cm}$ (Flores-Gallegos et al. 2019) con peso de 20 a $40 \mathrm{~kg}$ (Reyes-Valdés et al. 2019) e individuos uniformes con altura entre $1.50 \mathrm{y}$ $2.00 \mathrm{~m}$ (Olhagaray et al. 2004). Por lo tanto, la selección de caracteres morfológicos es el principio y el fundamento para la producción o aprovechamiento de sotol. Los resultados de la Figura 3 proporcionan información útil para el proceso de selección de plantas, de acuerdo con Cano et al. (2005) y Velasco et al. (2009) las variables altura de la planta, cobertura de roseta y diámetro de tallo pueden predecir adecuadamente el peso de la piña (tallo); lo que indica que estas variables son fundamentales para los estudios de aprovechamiento. Además, Rosales-Serna et al. (2020) también mencionan la importancia de estas variables para el crecimiento de la planta de sotol.

El empleo de herramientas digitales y de análisis en condiciones naturales es un campo que aún se encuentra en una etapa de desarrollo (Mishra et al. 2016). Las imágenes digitales se han uti- lizado mayormente en condiciones controladas, no obstante, la aplicación de estas herramientas en ambientes naturales aún es un desafío; ya que les variaciones y condiciones ambientales que ocurren en diferentes sitios y la falta de diseños e implementación de protocolos dificultan la captura y el análisis de los datos (Singh et al. 2015). El uso de fotografías y de análisis digital constituye una herramienta de apoyo para caracterizar las dimensiones morfológicas de las plantas simples de sotol en ambientes naturales. Además, proporciona información para la caracterización y selección de plantas a fin de un aprovechamiento adecuado en la producción de sotol. Este método no lleva mucho tiempo en la toma de fotografías y en el procesamiento de imágenes de un espécimen (5 a 10 minutos). Aunado a ello, una sola persona puede realizar todo el proceso, a diferencia de las mediciones manuales que puede llevarse más tiempo (15 a 20 minutos) y se necesita apoyo de otra persona. 
Adicionalmente, el método puede ser modificado para el uso de fotografías con drones, de tal forma que la cobertura de cantidad de individuos y de área de muestreo aumentará sustancialmente.

\section{AGRADECIMIENTOS}

Al Consejo de Ciencia y Tecnología (CONACYT) y a la Universidad Autónoma Agraria Antonio Narro (UAAAN) por su apoyo y financiamiento para esta investigación.

\section{LITERATURA CITADA}

Abbas A, Jain S, Gour M, Vankudothu S (2021) Tomato plant disease detection using transfer learning with CGAN synthetic images. Computers and Electronics in Agriculture 187: 1-9. DOI: 10.1016/j.compag.2021. 106279.

Acosta MM, Pérez MR, Romero SME, González HA, Martínez ÁL (2017) Estimación de la densidad forestal mediante imágenes Landsat ETM+ en la región sur del Estado de México. Revista Mexicana de Ciencias Forestales 8: 30-55.

Becerra-López JL, Rosales-Serna R, Ehsan M, Becerra-López JS, Czaja A, Estrada-Rodríguez JL, RomeroMéndez U, Santana-Espinosa S, Reyes-Rodríguez CM, Ríos-Saucedo JC, Domínguez-Martínez PA (2020) Climatic change and habitat availability for three sotol species in Mexico: a vision towards their sustainable use. Sustainability 12: 1-12. DOI: 10.3390/su12083455.

Breusch TS, Pagan AR (1979) A simple test for heteroscedasticity and random coefficient variation. Econometrica: Journal of the econometric society 47: 1287-1294.

Brophy JA, LaRue T, Dinneny JR (2018) Understanding and engineering plant form. Seminars in Cell \& Developmental Biology 79: 68-77.

Cano PA, Berlanga RCA, Castillo QD, Martínez BOU, Zárate LA (2005) Análisis dimensional y tablas de producción de sotol (Dasylirion cedrosanum Trel.) para el estado de Coahuila. Instituto Nacional de Investigaciones Agrícolas y Pecuarias - Centro de Investigación Regional Noreste (INIFAP-CIRNE). Folleto Técnico Núm. 18. Campo Experimental Saltillo. Saltillo, México. 29p.

Cano PA, Martínez BOU, Berlanga RCA, Villavicencio GEE, Castillo QD (2011) Guía para la evaluación de existencias de sotol (Dasylirion cedrosanum Trel.) en poblaciones naturales del estado de Coahuila. Instituto Nacional de Investigaciones Agrícolas y Pecuarias - Centro de Investigación Regional Noreste (INIFAPCIRNE). Folleto Técnico Núm. 43. Campo Experimental Saltillo. Coahuila, México. 24p.

CONABIO (2001) Comisión Nacional para el Conocimiento y Uso de la Biodiversidad. Portal de geoinformación sistema nacional de información sobre biodiversidad. http://www.conabio.gob.mx/informacion/gis/. Fecha de consulta: 15 de diciembre de 2021.

Cooksey RW (2020) Illustrating statistical procedures: Finding meaning in quantitative data. Third Edition. Springer Nature. Singapore. 737p.

Encina-Domínguez JA, Meave JA, Zárate-Lupercio A (2013) Structure and woody species diversity of the Dasylirion cedrosanum (Nolinaceae) rosette scrub of central and southern Coahuila State, México. Botanical Sciences 91: 335-347.

Ferreira T, Rasband W (2018) ImageJ userguide. IJ 1.52a Revised Edition. 186p. https://imagej.nih.gov/ij/docs/ guide/index.html. Fecha de consulta: 14 de diciembre de 2021. 
Flores-Gallegos AC, Cruz-Requena M, Castillo-Reyes F, Rutiaga-Quiñones OM, Torre LS, Paredes-Ortíz A, Soto ON, Rodríguez-Herrera R (2019) Sotol, an alcoholic beverage with rising importance in the worldwide commerce. Alcoholic Beverages 7: 141-160.

Gupta C, Tewari VK, Machavaram R, Shrivastava P (2021) An image processing approach for measurement of chili plant height and width under field conditions. Journal of the Saudi Society of Agricultural Sciences. DOI: 10.1016/j.jssas.2021.07.007.

Hassan MA, Yang M, Fu L, Rasheed A, Zheng B, Xia X, Xiao Y, He Z (2019) Accuracy assessment of plant height using an unmanned aerial vehicle for quantitative genomic analysis in bread wheat. Plant Methods 15: 1-12. DOI: 10.1186/s13007-019-0419-7.

IMPI (2002) Instituto Mexicano de la Propiedad Industrial. Declaración de protección a la denominación de origen sotol. https://www.gob.mx/impi/acciones-y-programas/declaraciones-generales-de-proteccion-de-denomina ciones-de-origen. Fecha de consulta: 25 septiembre 2021.

Kolhar S, Jagtap J (2021) Plant Trait Estimation and Classification Studies in Plant Phenotyping Using Machine Vision-A Review. Journal Information Processing in Agriculture. DOI: 10.1016/j.inpa.2021.02.006.

Kumar JP, Domnic S (2019) Image based leaf segmentation and counting in rosette plants. Information Processing in Agriculture 6: 233-246.

Lallana VH, Di Persia JF (2018) Caracterización morfométrica de semillas de cuatro especies de orquídeas terrestres nativas de Argentina. Ciencia, Docencia y Tecnología 29: 272-284.

Li C, Adhikari R, Yao Y, Miller AG, Kalbaugh K, Li D, Nemali K (2020) Measuring plant growth characteristics using smartphone based image analysis technique in controlled environment agriculture. Computers and Electronics in Agriculture 168: 1-8. DOI: 10.1016/j.compag.2019.105123.

Li Z, Guo R, Li M, Chen Y, Li G (2020) A review of computer vision technologies for plant phenotyping. Computers and Electronics in Agriculture 176: 1-21. DOI: 10.1016/j.compag.2020.105672.

Ma X, Zhu K, Guan H, Feng J, Yu S, Liu G (2019) Calculation method for phenotypic traits based on the 3D reconstruction of maize canopies. Sensors 19: 1-25. DOI: 10.3390/s19051201.

Mishra KB, Mishra A, Klem K, Govindjee (2016) Plant phenotyping: A perspective. Indian Journal of Plant Physiology 21: 514-527.

Olhagaray REC, Esparza CG, Vega SF (2004) Producción y comercialización de licores de sotol (Dasylirion cedrosanum Trel.) en Durango, México. Revista Mexicana de Ciencias Forestales 29: 83-89.

Perea-Flores MJ, Chanona-Perez JJ, Garibay-Febles, V, Calderon-Dominguez G, Terrés-Rojas E, MendozaPerez JA, Herrera-Bucio R (2011) Microscopy techniques and image analysis for evaluation of some chemical and physical properties and morphological features for seeds of the castor oil plant (Ricinus communis). Industrial Crops and Products 34: 1057-1065.

Reyes-Valdés MH, Benavides-Mendoza A, Ramírez-Rodríguez H, Villarreal-Quintanilla JA (2012) Biología e importancia del sotol (Dasylirion spp). Parte I: sistemática, genética y reproducción. Planta 7: 11-13.

Reyes-Valdés MH, Palacios R, Rivas-Martínez EN, Robledo-Olivo A, Antonio-Bautista A, Valdés-Dávila CM, Villarreal-Quintanilla JA, Benavides-Mendoza A (2019) The sustainability of mexican traditional beverage sotol: ecological, historical, and technical issues. Processing and sustainability of beverages 2: 103-137.

Rosales-Serna R, Ríos-Saucedo JC, Rosales-Mata S, Santana-Espinoza S, Domínguez-Martínez AP (2020) Fertilización química y crecimiento de maguey cenizo y sotol en plantaciones comerciales establecidas en Durango. Ciencia e Innovación 3: 43-52. 
SEMARNAT (1997) Norma Oficial Mexicana NOM-005-RECNAT-1997, Que establece los procedimientos, criterios y especificaciones para realizar el aprovechamiento, transporte y almacenamiento de corteza, tallos y plantas completas de vegetación forestal. http://www.dof.gob.mx/nota_detalle.php?codigo=4880104\&fecha =20/05/1997. Fecha de consulta: 25 septiembre de 2021 .

Singh, BD, Singh, AK (2015) Marker-assisted plant breeding: principles and practices. Springer, New Delhi. India. 514p.

Veerendra G, Swaroop R, Dattu DS, Jyothi C A, Singh MK (2021) Detecting plant diseases, quantifying and classifying digital image processing techniques. Journal Materials Today: Proceedings. DOI: 10.1016/j.matpr. 2021.06.271.

Velasco BE, Zamora MMC, Espinosa HP, Sampayo, CB, Moreno FS (2009) Modelos predictivos para la producción de productos forestales no maderables: Agaves mezcaleros. Centro Nacional de Investigación Disciplinaria en Conservación y Mejoramiento de Ecosistemas Forestales. Instituto Nacional de Investigaciones Agrícolas y Pecuarias. Manual Técnico Núm. 3. México. 60p.

Wang X, Singh D, Marla S, Morris G, Poland J (2018) Field-based high-throughput phenotyping of plant height in sorghum using different sensing technologies. Plant Methods 14: Article number 53. DOI: 10.1186/s13007018-0324-5.

Wei H, Zhao Y, Xie Y, Wang H (2018) Exploiting SPL genes to improve maize plant architecture tailored for high-density planting. Journal of Experimental Botany 69: 4675-4688.

Zhang W (2020) Digital image processing method for estimating leaf length and width tested using kiwifruit leaves (Actinidia chinensis Planch). PloS One 15: e0235499. DOI: 10.1371/journal.pone.0235499.

Zhou S, Chai X, Yang Z, Wang H, Yang C, Sun T (2021) Maize-IAS: a maize image analysis software using deep learning for high-throughput plant phenotyping. Plant Methods 17: Article number 48. DOI: 10.1186/s13007021-00747-0. 